\title{
Advantages of Simultaneous Imaging Using an Atomic Force Microscope Integrated with an Inverted Light Microscope
}

\section{W. Travis Johnson}

Agilent Technologies, Nanotechnology Measurements Division

4330 West Chandler Blvd, Chandler, AZ 85226

w-travis_johnson@agilent.com

\section{Introduction}

Atomic Force Microscopy (AFM) permits measurements on biological samples below the limits of light microscopy resolution under physiological environments and other controlled conditions [1]. Consequently, AFM has become an increasingly valuable technique in cell biology [2]. One of the most exciting advances in AFM instrumentation has been its integration with the light microscope [3]. This permits investigators to take advantage of the power and utility of light microscopy and scanning probe microscopy simultaneously. In combining a light microscope with an AFM, scanner components must be specifically designed so that they do not adversely impact the light microscope's optical imaging capabilities. For example, an AFM-ILM (inverted light microscope) hybrid system should be fully compatible with the highest quality, off-the-shelf $0.50-0.55 \mathrm{NA}$ numerical aperture (NA) OEM objectives and condensers.

An example of an ILM-AFM system that does not compromise AFM performance, nor interfere with the ILM's imaging capabilities, is Agilent's 6000ILM AFM, which is pictured in Figure 1. This instrument seamlessly integrates the high-resolution AFM with the researcher's choice of light microscope: Zeiss Axio Observer (A1, D1, or Z1), Nikon Ti, or Olympus IX series 71/81.

\section{Methods and Materials}

Instrumentation. With the AFM in place, the ILM-AFM is capable of operating in the most popular and effective light microscopy modes, including bright field (Kohler) illumination, differential interference contrast (DIC), phase contrast, fluorescence, and even confocal imaging. Thus, translucent cells and other semi-transparent samples can be easily located with the ILM and further investigated at higher resolutions with the AFM. The ILM-AFM also supports the most popular and useful AFM imaging modes, including contact mode, acoustic AC (AAC) mode [4], magnetic AC (MAC) mode [5], and topography and recognition imaging (TREC) [6]. TREC, which is not directly addressed further in this article, is a useful technique that maps molecular interactions, much like force-volume spectroscopy [7], albeit with much greater speed and better resolution.

Separate $X-Y$ and $Z$ AFM scanners eliminate horizontalvertical crosstalk, which can occur when $X-Y$ and $Z$ piezos are combined in the same scanner. Closed-loop sample scanning stages that provide at least $100 \times 100 \mu \mathrm{m}$ of range in the horizontal plane are necessary for cell imaging because they minimize the impact of hysteresis and permit extremely accurate positioning and repositioning of samples under the AFM probe. An unobstructed optical path through the $Z$ scanner avoids any need for a complicated system of lenses and mirrors, which can be detrimental to high-quality light microscopy applications. A closed-loop $Z$ scanner with a large $Z$ range (for example $30 \mu \mathrm{m}$ ) is also an important feature because it permits the ILM-AFM to be compatible with the relatively large sample sizes that are often encountered with cultured cells and tissue samples.

Sample Formats. Hybrid ILM-AFMs should accommodate sample formats that are commonly used in cell and tissue imaging applications in both air and in-liquid environments. These include sample plates for $1 \times 3$ inch microscope slides, 35 and $50 \mathrm{~mm}$ Pitre dishes, and $22-25 \mathrm{~mm}$ glass cover slips. Other very useful features for prolonging cell viability are sample plates that are able to maintain cells at physiological temperature $\left(37^{\circ} \mathrm{C}\right)$ and maintain optimal cell culture atmospheres (for example, $5 \% \mathrm{CO}_{2}$ ) [8].

Specimens and Data Acquisition. Data and images for the T24 and MyEND cells were obtained in professor Peter Hinterdorfer's group at the Institute for Biophysics University of Linz, Linz, Austria. The other cells used in this study were

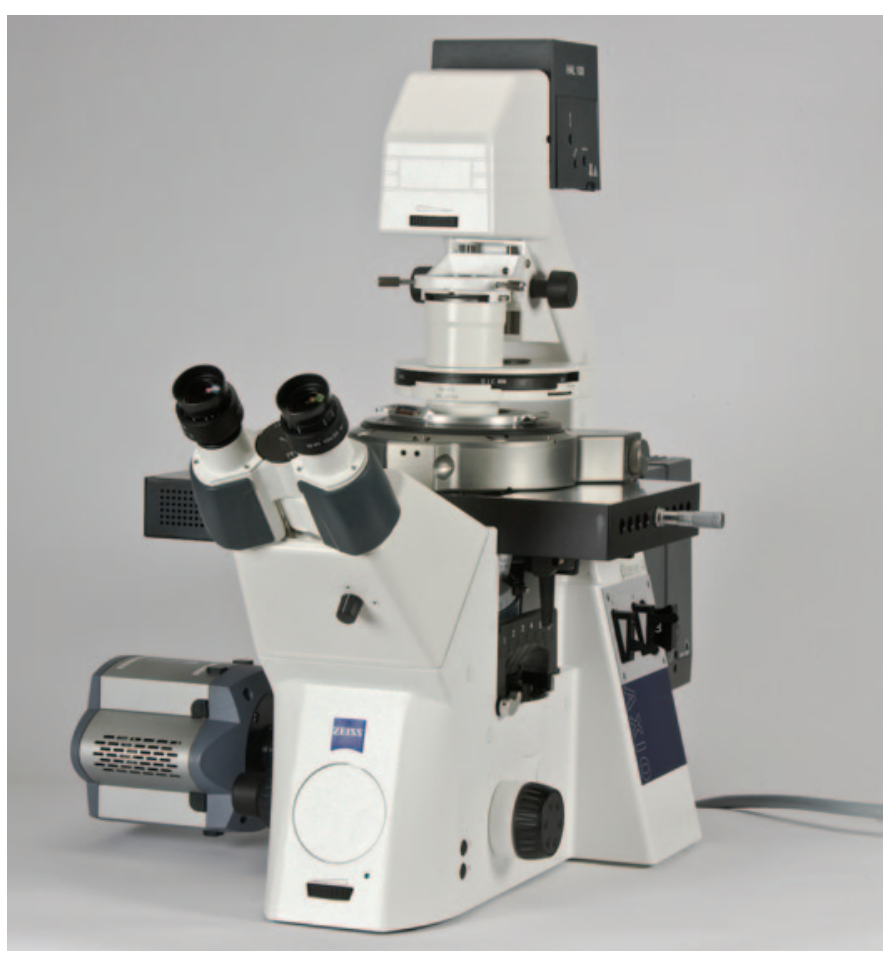

Figure 1: An example of an AFM that was designed specifically for combined ILM-AFM studies; the Agilent 6000ILM AFM mounted on a Zeiss D1 ILM. 


\section{the highest quality...}

\section{the most precise sectioning... incomparable durability}

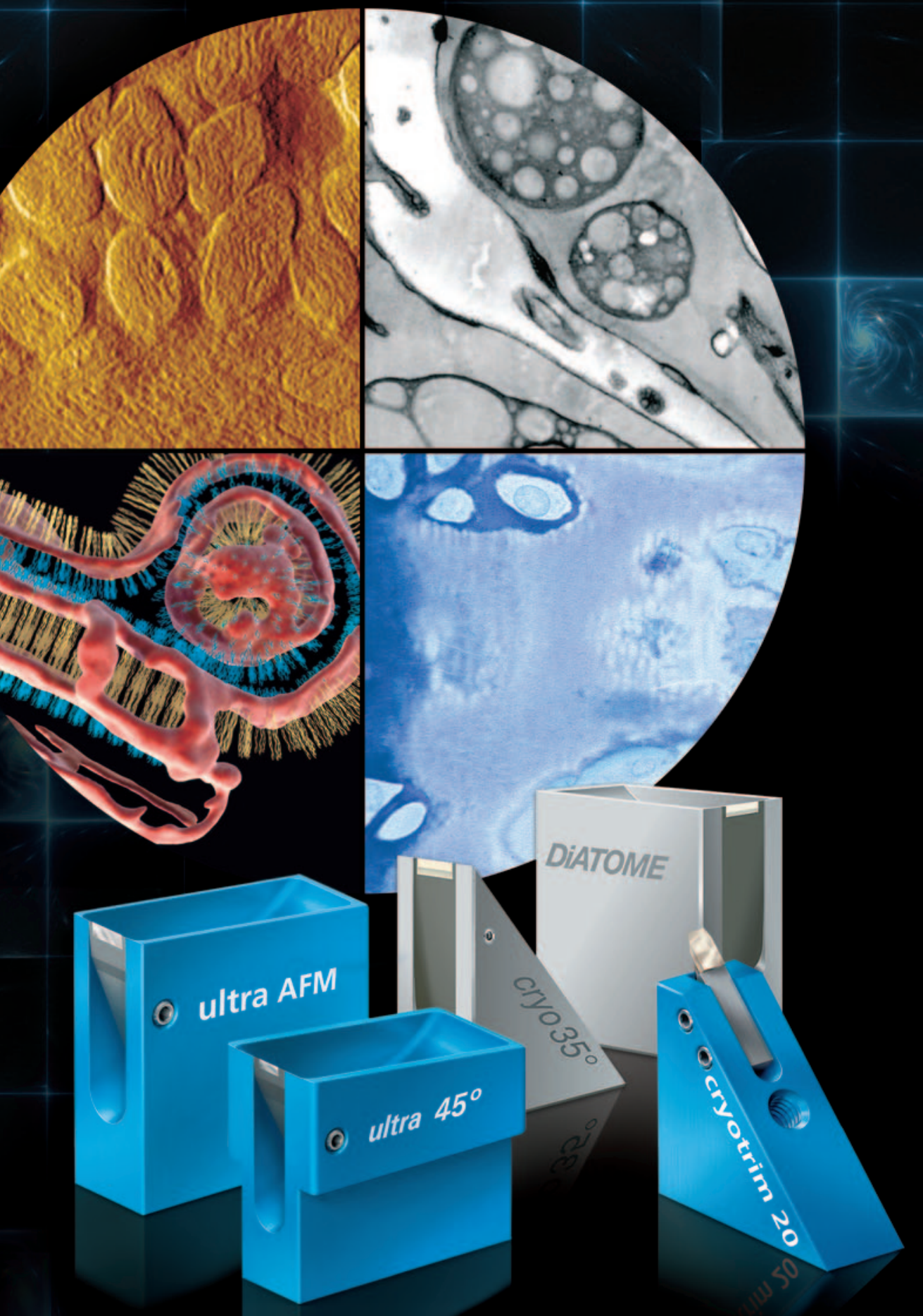

\section{Free Customer Service}

Sectioning tests with biological and material research specimens of all kinds. We send you the sections along with the surfaced sample, a report on the results obtained and a recommendation of a suitable knife. Complete discretion when working with proprietary samples.

\section{Re-sharpening and Reworking Service}

A re-sharpened Diatome diamond knife demonstrates the same high quality as a new knife. Even knives purchased in previous years can continue to be resharpened. The knives can be reworked into another type of knife for no extra charge, e.g. ultra to cryo or $45^{\circ}$ to $35^{\circ}$.

\section{Exchange Service}

Whenever you exchange a knife we offer you a new Diatome knife at an advantageous price.

40 years of development, manufacturing, and customer service

\section{DIATOME} diamond knives

ultra $45^{\circ} \bullet$ cryo $\bullet$ histo $\bullet$ ultra $35^{\circ} \bullet$ STATIC LINE II cryo-P • cryo immuno $\bullet$ ultra sonic cryotrim 45 and 25 ultra $\bullet$ AFM \& cryo AFM • cryo $25^{\circ}$ 
gifts from professor Tatiana P. Ugarova and Ivan Yermolenko of Arizona State University. The cells were grown at $37^{\circ} \mathrm{C}$ at $5 \% \mathrm{CO}_{2}$, on bare glass cover slips or protein coated cover slips in cell-specific culture media. In some cases, the cells were lightly fixed by adding low concentrations of glutaraldehyde to the medium. Fluorescently labeled normal human cells were stained with phalloidin-Alexa568. All AFM experiments were performed using an Agilent 6000ILM AFM on a Zeiss Axio Observer that was equipped with phase contrast, DIC, and fluorescence as well as a $0.55 \mathrm{NA}$ condenser. Cover slips containing the cells were mounted onto a perfusion cell sample holder or a standard liquid cell. The laser was aligned on the AFM probes manually via software control. The sample holder was filled with PBS buffer, and the photo detector was aligned using the automated alignment feature in PicoView. For MAC, and AAC mode imaging, MAC Levers were tuned to resonance using the auto tune feature. The cells were imaged at room temperature in either contact, AAC or MAC mode as described in the figure legends. The "Point and Shoot" feature was used to position areas of interest under the tip of AFM probes to enable precise AFM imaging at the predefined locations indentified by the light microscope (bright field, DIC, phase contrast, and/ or fluorescence). The overlay feature was used to overlay the fluorescent images onto the AFM images.

Figure $2 \mathrm{a}$ shows a $40 \times$ DIC optical image of living cells derived from human cervical cancer cells (HeLa) [9]. The red box indicates the area from which an AFM image is to be acquired using "Point and Shoot." In Figure 2b, the AFM contact-mode deflection image was overlaid on the optical image from $2 \mathrm{a}$ in real time. Figure $2 \mathrm{c}$ is a $3 \mathrm{D}$ contact-mode AFM image of cells in (b). The deflection image was overlaid on the 3D topography image using PicoImage software in (c) to show both the depth and finer details of the cells. The cells were grown on collagen-coated cover slips and imaged in HEPES buffer containing BSA.

\section{Applications}

Differential interference contrast and phase contrast light microscopy techniques [10] permit even semi-transparent samples to be visualized and located so that they can be investigated with the AFM. An example of DIC combined with AFM imaging is presented in Figure 3. A normal cell line (WI38) originating from human lung tissue [11] can be seen in the optical image as well as in the AFM image; they have a fibroblast-like morphology. In this case, a WI38 cell was identified by DIC (Figure 3b) so the sample could be positioned directly under the tip of the AFM probe. The cell was then imaged in PBS buffer using contact-mode AFM (Figure 3a).

Mouse vascular endothelial (MyEND) cells [12] and other vascular cells contain particular membrane-bound structures, called Weibel-Palade bodies (WPB). WPBs are storage organelles for von Willebrand factor (VWF), which is a glycoprotein that mediates the adherence of platelets to one another and to sites of vascular damage [13]. In this manner VWF promotes blood clot formation leading to wound healing. Bumps on the vascular endothelial cell surface observed in high-resolution AFM images may be indicative of the presence of intact WPBs located under the plasma membrane. WPB secretion pores can also form on the surface of vascular endothelial cells. The pores often indicate fusion events between WPBs and the plasma membrane, leading to
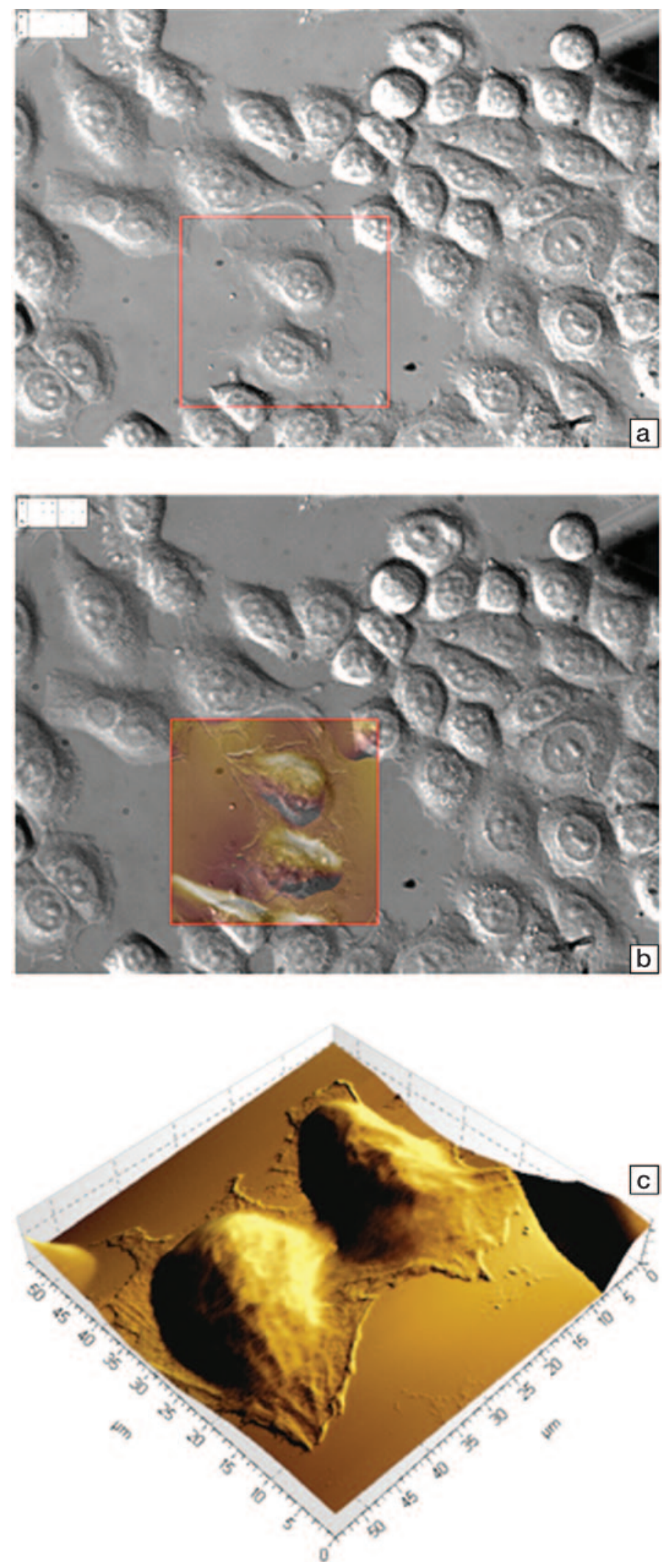

Figure 2: (a) 40× DIC optical image of living cells derived from human cervical cancer cells (HeLa). The red box indicates the area from which the AFM image is to be acquired using "Point and Shoot." (b) Contact-mode deflection image $(60 \times 60 \mu \mathrm{m})$ overlaid on the area in the red box indicated in $2 a$. (c) 3D contact-mode topography image combined with the deflection image of the cells in (b) $(60 \times 60 \mu \mathrm{m})$. 


\section{Finally, Scientific CMOS for everyone - at a fraction of the cost!}

QImaging Introduces the Rolera ${ }^{\mathrm{TM}}$ Bolt

Take advantage of all the latest features

in this new camera. The Rolera Bolt is

easy to purchase, easy to install and

easy to use. Discover how you and

your team can implement Scientific

CMOS today - for nearly half the price!

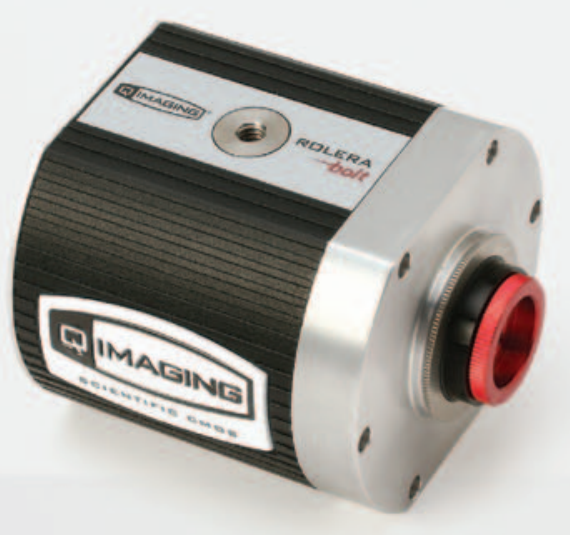

R日LERA bolt

\section{Scientific CMOS}

Camera from QImaging

Easily achievable for every research facility, including yours.
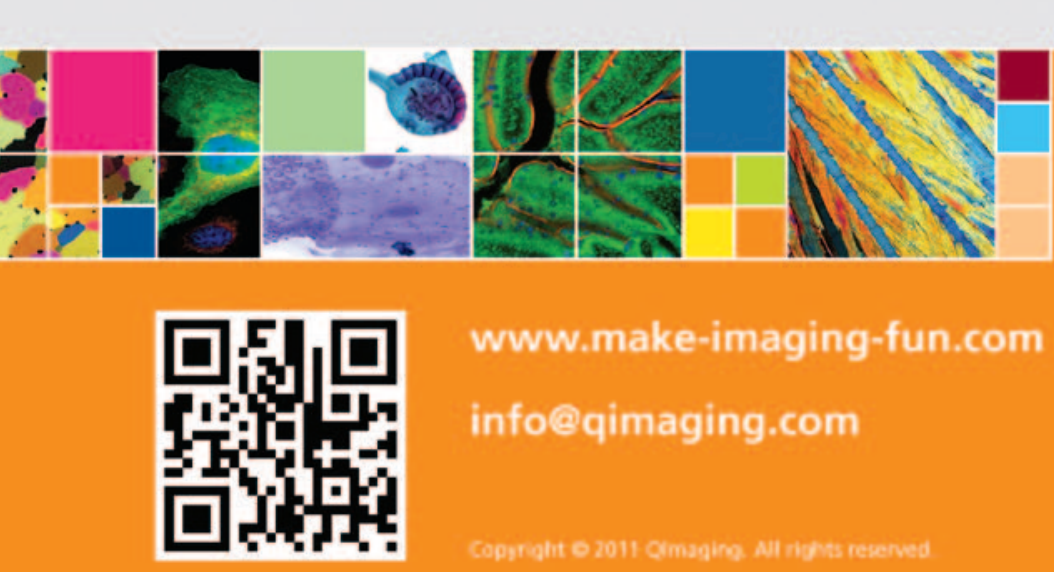

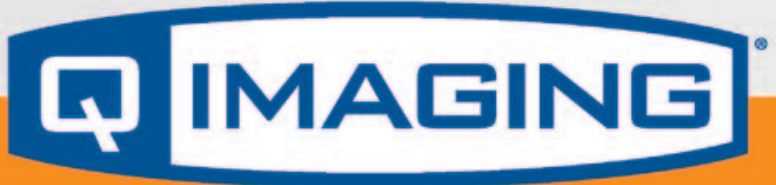

DIGITAL IMAGING MADE EASY 

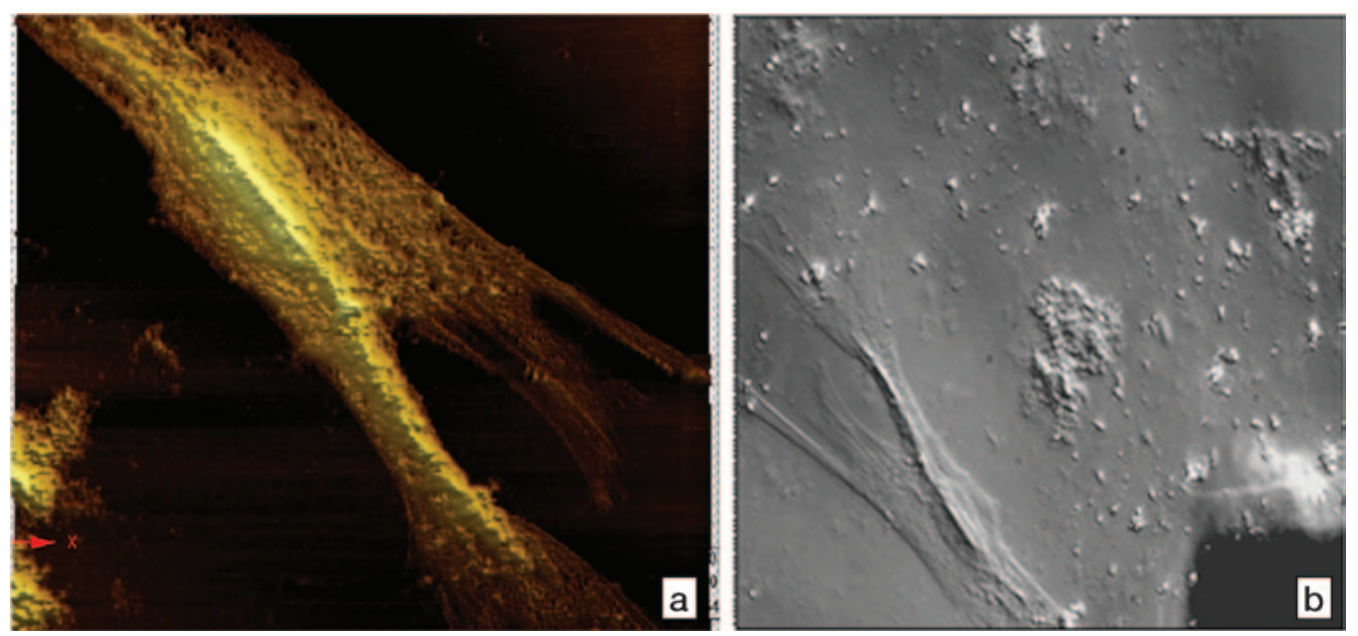

Figure 3: Normal human lung (WI38) cell. These cells display a fibroblast-like morphology that is clearly evident in the AFM and DIC optical images. (a) 3D contact-mode AFM topography image $(90 \times 90 \mu \mathrm{m})$. (b) Corresponding 40× DIC optical image of the same cell (image width $=120 \mu \mathrm{m}$ ).
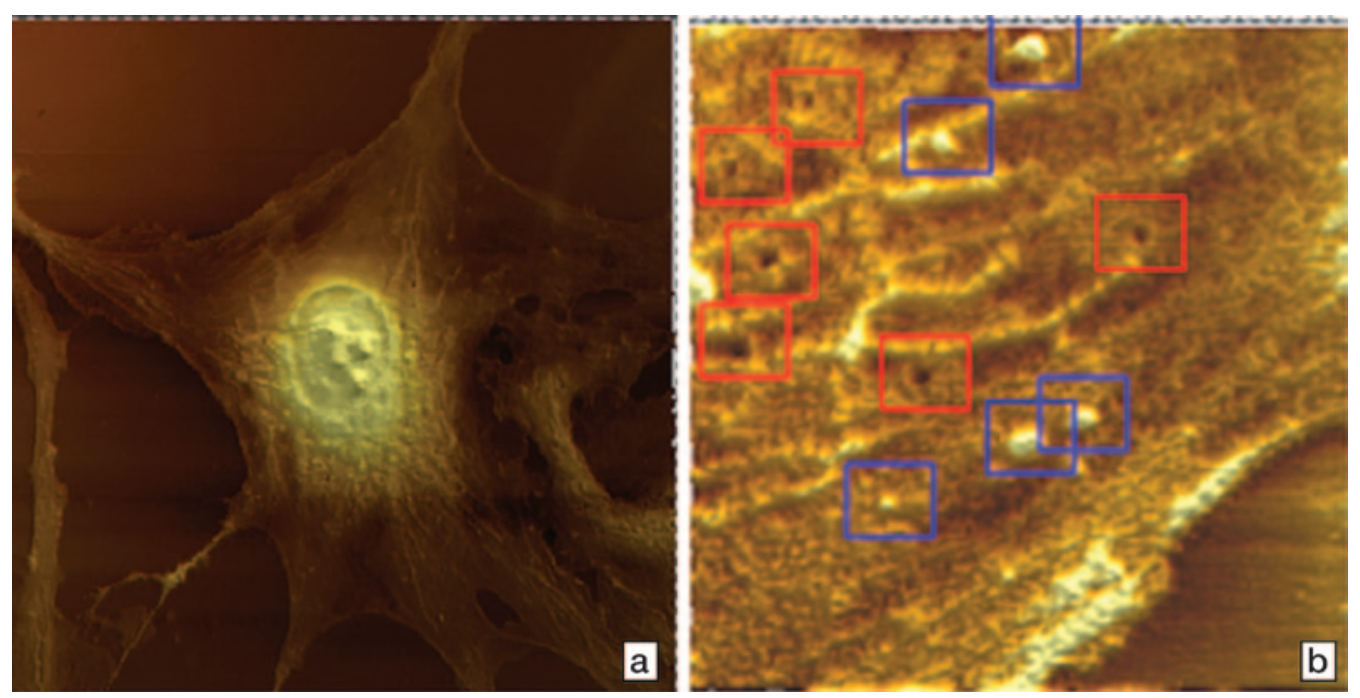

Figure 4: 3D contact-mode AFM images of a MyEND cell. (a) Contact-mode AFM topography image $(70 \times 70 \mu \mathrm{m})$. In (b), a feature of interest that was found in image (a) was repositioned under the AFM probe and imaged at higher resolution $(14 \times 14 \mu \mathrm{m})$. Inside the blue squares, small humps may be indicative of the presence of WPBs. The pore-like indentations in the red squares may indicate fusion events between the plasma membrane and WPBs leading to exocytosis of VWF (see text for further explanation).

exocytosis of VWF following hypoxic stimulation or vascular injury. In the high-magnification contact-mode AFM image shown in Figure 4b, both humps (blue squares) and pores (red squares) can clearly be observed. Because the WPBs are on the order of several hundred nanometers in diameter, the humps and the pores are too small to be easily resolved by standard light microscopy techniques.

Using an ILM-AFM outfitted with fluorescence microscopy capabilities permits combined AFM-fluorescence microscopy studies [3]. The AFM can often resolve small structures that cannot be easily or efficiently labeled with fluorescent probes. Figure 5 shows a specific example of a combined AFM-fluorescence microscopy application using WI38 cells. In this case, the cells were grown on fibronectin-coated glass cover slips and fluorescently labeled with Alexa568 conjugated to a phallotoxin (phalloidin-Alexa568), which tightly and specifically binds to actin filaments [14]. A cell was located in the optical view using DIC along with fluorescence microscopy, and then it was positioned precisely under the tip of the AFM probe using "Point and Shoot." The cell was then imaged in contact mode as shown in figure 5 . The fluorescence image of the cell is shown in figure $5 \mathrm{a}$. The yellow box indicates the area from which the AFM $75 \times 75 \mu \mathrm{m}$ contact-mode AFM image was acquired. The fluorescence image was acquired using a Lumen Dynamics X-CITE 120Q fluorescence light source, an $\mathrm{LD}$ Plan-Neofluar 40× objective, and a $63 \mathrm{HE}$ mRFP shift-free filter set.

Lipid rafts serve as organizing centers for the assembly of signaling molecules. They also influence membrane fluidity, receptor trafficking, as well as other protein trafficking events. The ILM-AFM permitted investigation of the morphology of live T24 (human bladder carcinoma) cells and the distribution of the lipid rafts. T24 cells were transfected to express green fluorescent protein (GFP) in the lipid rafts [15]. The "Point and Shoot" capability of the software and the motorized sample stage allowed for rapid screening of the sample surface for suitable cells. The cell positions were located using DIC imaging (Figure 6a). GFP expression levels were determined by fluorescence intensity (Figure 6b). The brightest areas indicate locations where GFP was over-expressed. The distribution of the lipid rafts was determined by changing the focal plane of the fluorescence images. For topographical imaging, a protein-expressing cell that was surrounded by non-fluorescent cells was selected (red square in Figure 6a) and imaged in AAC mode. The filaments of the cytoskeleton are clearly resolved, as are lamellipodia. Several small connections between individual cells can also be observed. An overlay of the fluorescence and the topography image (Figure 6d) allowed the position of GFP-labeled lipid rafts to be correlated with topographical features such as cytoskeleton filaments and lamellipodia.

In Figure 7, the locations of lipid rafts and cytoskeletal elements in T24 cells were correlated using fluorescence microscopy. The GFP fluorescence image (Figure 7a) represents the distribution of lipid rafts, most of which were found near 


\section{The Helium Ion Microscope Beyond Gallium and Electrons}

High Resolution Surface Imaging I Insulating Samples Imaging 3D Nanofabrication | Thin Film Analytics

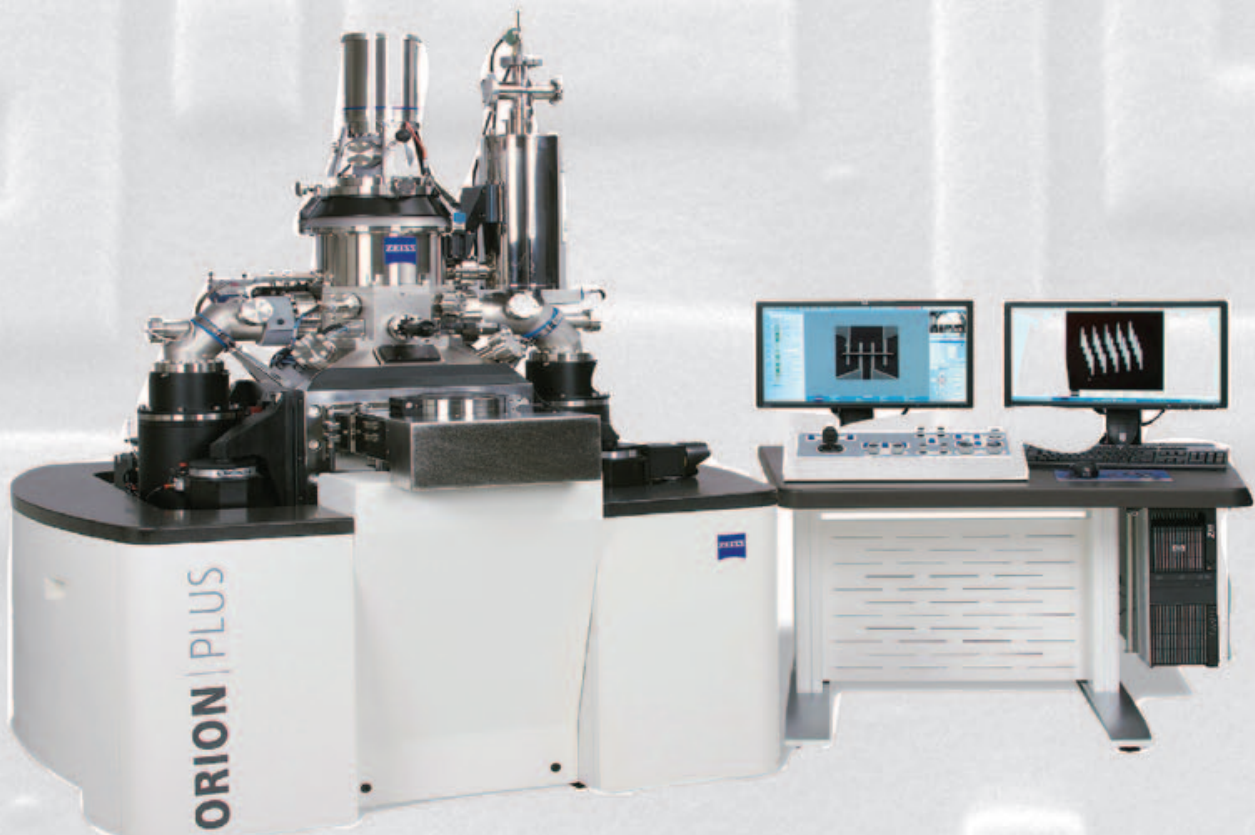

Key Features:

- $<0.35 \mathrm{~nm}$ probe size for high resolution surface imaging with high depth of field

- Superior charge control allows easy imaging of insulating samples without conductive coating

- Gas-assisted material deposition and helium ion milling for precise nanofabrication

- RBS like analysis at the nanoscale

Visit us at MRS - Booth \#501

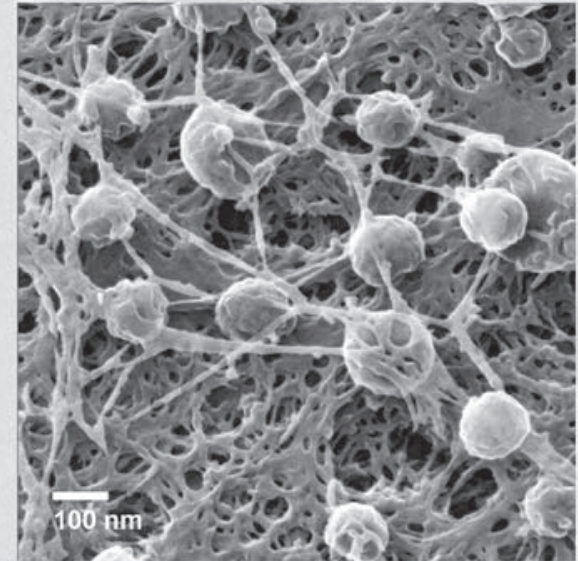

High-resolution image of an uncoated biological sample showing virus particles attached to a cell.

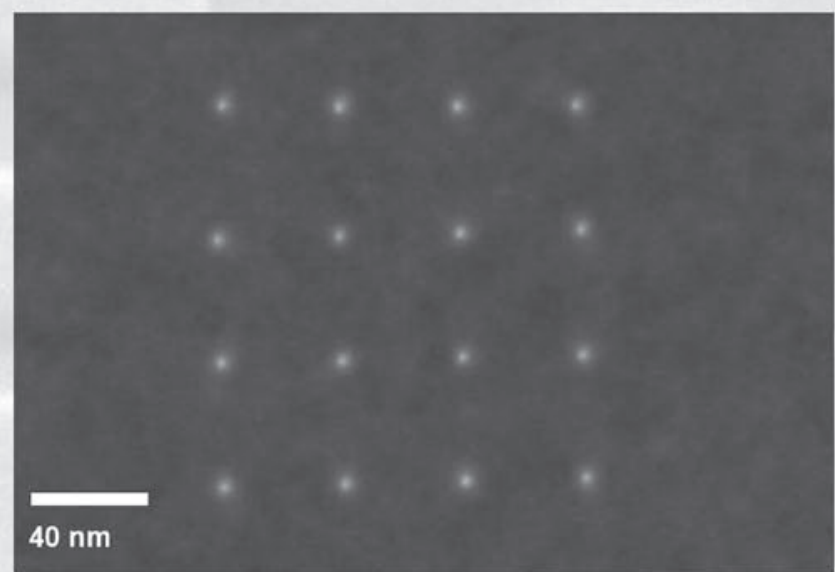

Array of $5( \pm 1) \mathrm{nm}$ nanopores in $\mathrm{Si}_{3} \mathrm{~N}_{4}$ membrane. A $10 \times 10$ array can be created in under one minute.

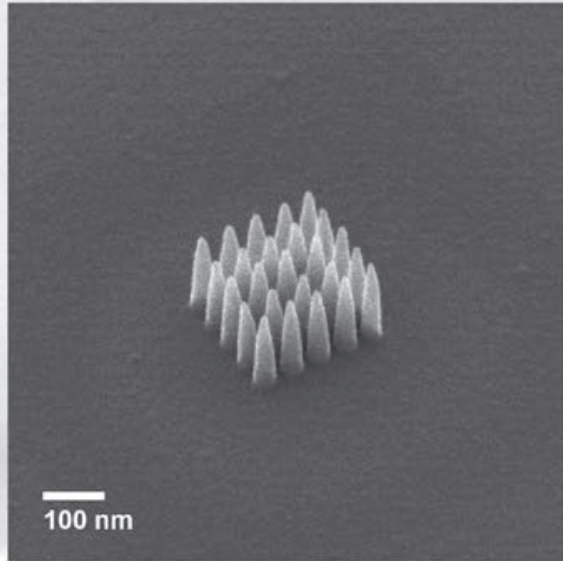

Array of Pt nano-pillars ( $35 \mathrm{~nm}$ diameter \& $50 \mathrm{~nm}$ pitch) deposited using the helium ion beam.
Carl Zeiss NTS

Nano Technology Systems
Carl Zeiss NTS, LLC One Corporation Way Peabody, MA 01960 U.S.A.
Tel: $978-826-1500$

Fax: 978-532-2503

info-usa@nts.zeiss.com www.zeiss.com/nts

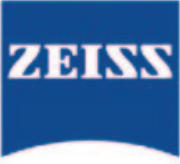

We make it visible. 

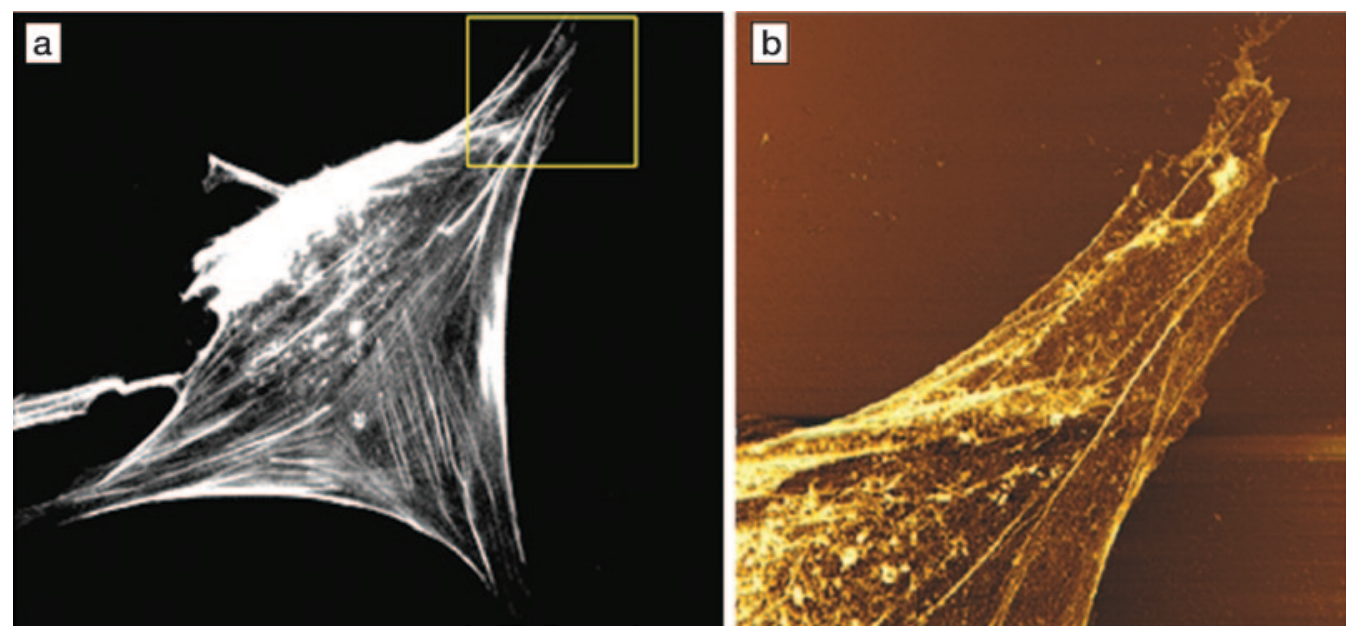

Figure 5: AFM and fluorescence images of WI38 cells that were labeled with phalloidin-Alex568 to highlight the actin filaments. (a) The fluorescence image (image width $=300 \mu \mathrm{m})(\mathrm{b}) 75 \times 75 \mu \mathrm{m}$ 3D contact-mode AFM topography image, which corresponds to the area indicated inside the yellow box in (a).

the cell nucleus. In addition, the cytoskeletal filaments were also stained with a fluorescent dye conjugate, phalloidinrhodamine, which is a red fluorescent stain that binds to actin filaments. Several of the filaments appeared to be pointed directly from the nucleus to the cell border (Figure $7 \mathrm{~b}$ ). Overlaying the GFP fluorescence image with the rhodamine
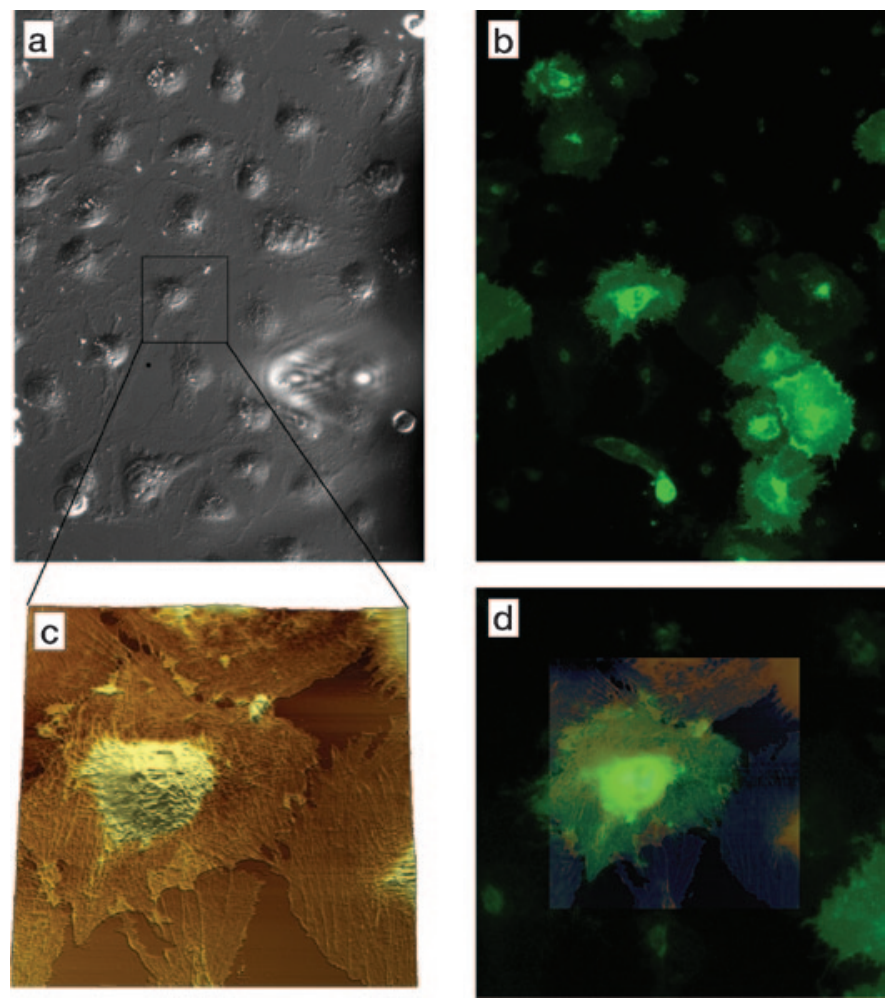

Figure 6: DIC, fluorescence, and AFM images of living T24 cells that were transfected to express GFP in their membranes. (a) DIC optical image of the T24 cells. (b) Lipid raft distribution measured by GFP fluorescence of the same area as in (a). (c) 3D AAC mode topography image $(90 \times 90 \mu \mathrm{m})$ of a GFP protein expressing cell interacting with other cells that do not over-express GFP. The cytoskeleton is clearly resolved and connections between individual cells are also visible. (d) Overlay of the topography and fluorescence images. Images and data courtesy of Institute for Biophysics, University of Linz, Austria. fluorescence image (Figure 7c) indicated a high degree of spatial correlation between the lipid rafts and the cytoskeletal elements (correlation coefficient 0.85 ). The 3D topographical image (Figure $7 \mathrm{~d}$ ) indicates that the dimensions of the T24 cells are approximately $4 \mu \mathrm{m}(Z)$ by $60 \mu \mathrm{m}(X-Y)$.

\section{Conclusions}

Many biological studies require more capabilities than either a light microscope or a stand-alone AFM can provide. By combining the AFM with an inverted light microscope to form a hybrid ILM-AFM system, some inherent limitations of AFM and light microscopy can be minimized without losing the favorable attributes of these methods. Open scanner architectures do not obstruct the optical path to the sample and permit the use of off-the-shelf, high-NA
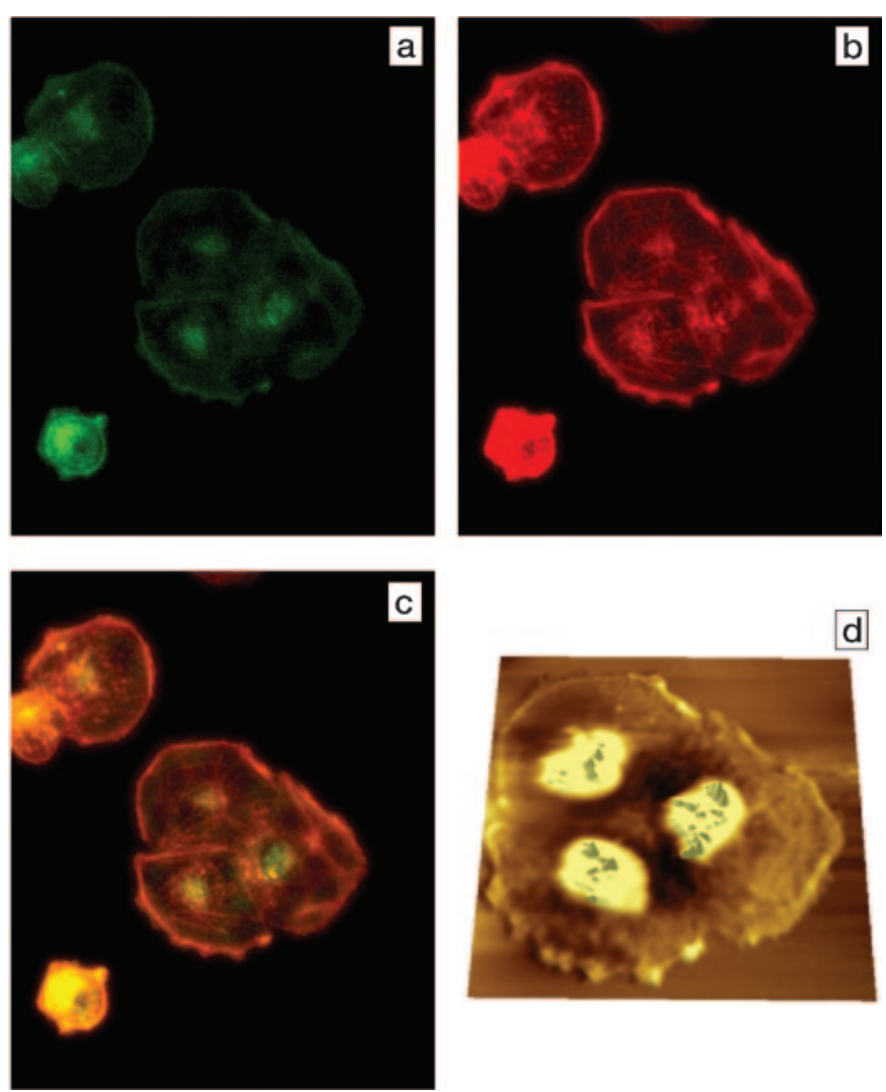

Figure 7: Phalloidin-rhodamine stained T24 cells that also express GFP in lipid rafts. (a) The GFP fluorescence channel demonstrates the distribution of the lipid rafts in the cells. (b) The rhodamine fluorescence channel shows the location of actin filaments in the same cells. (c) A rhodamine/GFP fluorescence overlay image was used to correlate the positions of the lipid rafts in relation to the cytoskeleton. (d) 3D MAC mode topography image of the same cluster of cells $(90 \times 90 \mu \mathrm{m})$. Images and data courtesy of Institute for Biophysics, University of Linz, Austria. 
condensers, and other high-quality optical components. Differential interference contrast, phase contrast imaging, or fluorescence microscopy can be used to expedite the identification of individual cells and features of interest. When combined with "Point and Shoot" capabilities, the optical images can be used to quickly and precisely position samples directly under the tip of an AFM probe.

\section{References}

[1] Nowakowski et al., Biochimica et Biophysica Acta 1514(2) (2001) 170-76.

[2] Goldsbury et al., Current Protocols in Protein Science (58) (2009) 171-217.

[3] Madl et al., Ultramicroscopy 106 (2006) 645-51.

[4] Schmitz et al., Anal Chem 69(6) (1997) 1012-18.

[5] Han and Lindsay, Appl Phys Lett 72 (1998) 1656.

[6] Stroh et al., PNAS 101 (2004) 12503-12507.

[7] Gaboriauda et al., Colloids and Surfaces B: Biointerfaces 62(2) (2008) 206-13.

[8] Baudin et al., Nat Protoc 2(3) (2007) 481-485.

[9] Masters, Nature Reviews Cancer 2 (2002) 315-319.

[10] Frohlich, J Vis Exp 17 (2008) 844.

[11] Wolosewick and Porter, American Journal of Anatomy 149(2) (1977) 197-225.

[12] Adamson et al., Journal of Physiology 539 (2002) 295-308.

[13] Metcalf et al., J Cell Sci 1(121) (2008) 19-27.

[14] Vandekerckhove et al., EMBO J 4(11) (1985) 2815-18.

[15] Weghuber et al., Anal Bioanal Chem 397(8) (2010) 3339-47.

MT
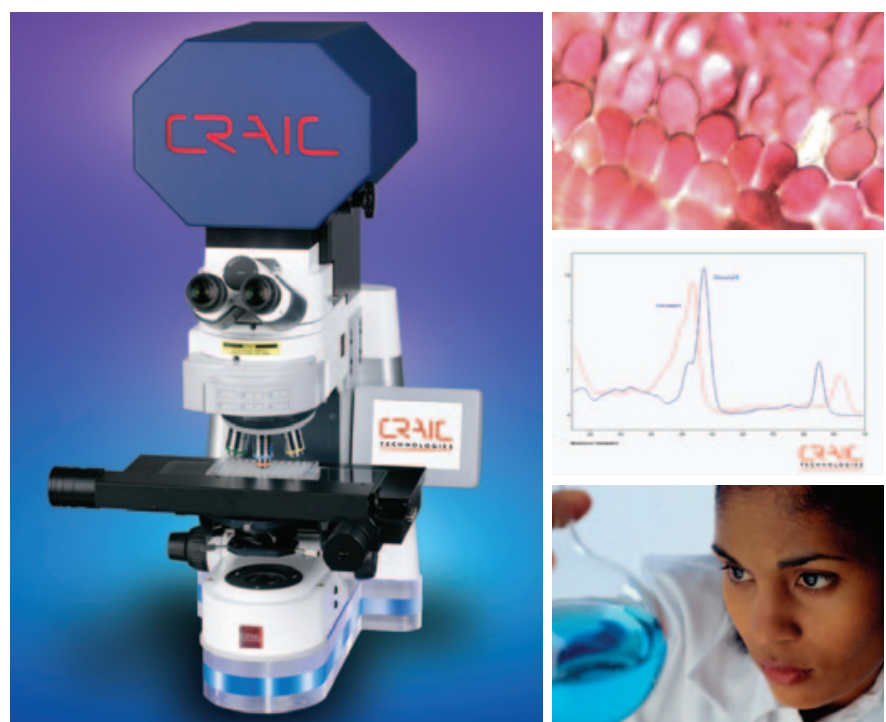

SPECTROSCOPY OF MICROSCOPIC SAMPLES

CRAIC Technologies UV-visible-NIR microscopes and microspectrophotometers are used for imaging and spectral analysis of sub-micron sized features with absorbance, reflectance, fluorescence, emission and polarized illumination. Capabilities include film thickness measurements, colorimetry and high resolution imaging in the UV, visible and NIR regions. Rapid \& accurate spectra \& images of microscopic samples: The Perfect Vision for Science ${ }^{\mathrm{TM}}$.

For more information, call 877.UV.CRAIC or visit our website at www.microspectra.com @2011 CRAIC Technologies, Inc. San Dimas, California (USA).

\section{Mini-SEM}

\section{www.Mini-SEM.com}

\section{Tabletop Scanning Electron Microscope}

Magnify Organic and Inorganic Samples $10 \mathrm{X}$ to $30,000 \mathrm{X}$

Elemental Identification \& Concentration (5-B to 92-U)

Elemental Mapping

Particle Counting and Sizing

Sample Preparation - None or Limited

Installation \& Training in Minutes

\section{Call for Special Educational Pricing}
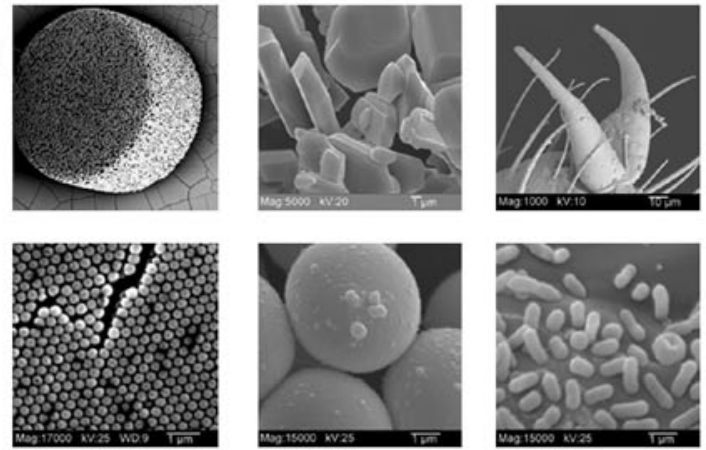